\title{
13. \\ Bemerkungen über die Bildung der Primzahlen aus einander.
}

( Vom Herrn Prof. H. F. Scherk in Halle,)

Eine geometrische, mit der Theorie der Primzahlen scheinbar nur in sehr entfernter Verbindung stehende Untersuchung veranlafste mich vor längerer Zeit zu dem Versuche, die Primzahl 17 aus allen kleineren Primzahlen und der Zahl 1 auf möglichst einfache Weise zusammenzusetzen. Hier ergab sich denn bald, dafs $17=1+2-3-5+7-11+2.13$, d, h. dafs 17 durch blofse Addition und Subtraction aus allen kleineren Primzahlen, wenn die Zahl 1 der Kürze halber mit zu diesen gerechnet wird, zusammengesetzt werden könne, wobei jedoch die nächstvorhergehende Primzahl 13 zweimal genommen werden müsse. Dies führte zu der Vermuthung, dafs dieselbe Eigenschaft vielleicht allen Primzahlen von der Form $4 n+1$ zukommen möchte. Aber diese Vermuthung bewährte sich nicht; denn es ist z. B. $13=1+2-3-5+7+11$, so dafs die der 13 nächstvorhergehende Primzahl blofs Einmal genommen zu werden braucht, dahingegen $19=1-2+3+5-7-11+13+17$ und $23=1-2+3-$ $5+7+11-13-17+2.19$. Da nun 13 wie 17 von der Form $4 n+1$, 19 wie 23 von der Form $4 n+3$ sind, das Bildungsgesetz aber bei keiner in diesen beiden Paaren sich befindenden Primzahl in der Hinsicht dasselbe bleibt, ob die nächstyorhergehende Primzahl einfach oder doppelt genommen werden mufs, so bot sich die Idee dar, von der F orm der Primzahlen ganz zu abstrahiren, und vielmehr auf die Stelle zu sehen, welche sie in der natïrlichen Reihe der Primzahlen einnehmen, dạ unter den aufeinander folgenden Primzahlen 13, 17, 19, 23 dasselbe Bildungsgesetz für 13 und 19, und für 17 und 23 Statt findet.

Auf diese Weise gelangte ich durch eine nicht bewiesene Induction zu folgenden Sätzen. Trennt man in der natürlichen Reihe der Primzahlen 2, 3, 5, 7, 11, 13 etc. die in ungerader Stelle stehenden $2,5,11$ etc. von den in gerader Stelle stehenden 3, 7, 13 etc., so kann Crelle's Jonrnal d. M. Bd.X. Hft. 3. 
Erstens. Jede geradstellige Primzahl aus allen kleineren und der Zahl 1 durch blofse Addition und Subtraction, so dafs jede von ihmen nur Einmal genommen wird, zusammengesetzt werden.

$\mathbf{Z}$ eitens. Jede ungeradstellige Primzahl kann aus allen kleineren und der Zahl 1 auf dieselbe Weise gebildet werden, mit dem Unterschiede jedoch, dafs die nächstvorhergehende Primzahl doppelt genommen werden mufs.

Was hierbei aufser der einfachen Bildungsart besonders merkwürdig scheint, ist der, so viel mir bekannt ist, sonst noch nirgends hervorgetretene Unterschied zwischen denjenigen Primzahlen, die in der natürlichen Reihe aller Primzahlen eine gerade, und derjenigen, die in derselben Reihe eine ungerade Stelle einnehmen. Dals keine Primzahl der einen Art nach dem Formationsgesetz der anderen Art gebildet werden könne (die Zahl 3 ausgenommen, auf welche wir jedoch sogleich besonders zurückkommen werden), liegt am Tage. Denn jeder geradstelligen Primzahl geht 1 und eine ungerade Menge von Primzahlen vorher; da sich nun unter diesen die einzige gerade Zahl 2 findet, so ist klar, dafs die algebraische Summe aller dieser Zahlen, wenn sie nach Belieben positiv oder negativ genommen werden, stets eine ungerade Zahl sein wird, und folglich auch vielleicht die verlangte Primzahl sein kann; wenn aber eine von ihnen, die von der 2 verschieden ist, doppelt, oder überhaupt eine gerade Anzahl mal genommen wird, so ist das Resultat eine gerade Zahl, und kann folglich der verlangten Primzahl nicht gleich sein. Demnach kann keine geradstellige Primzahl nach dem zweiten Satze gebildet werden. Ganz auf dieselbe Weise läfst sich zeigen, dafs keine ungeradstellige Primzahl nach dem ersten Satze zusammengesetzt werden kann. Dieses Raisonnement ist aber offenbar dann nicht anwendbar, wenn diejenige Primzahl, welche eine gerade Anzahl mal genommen wird, die 2 ist; und demnach ist es möglich, dafs die Primzahl 3 , bei deren Formation 2 die einzige in Betracht kommende Primzahl ist, sowohl nach der einen, als nach der andern Regel gebildet werden kann, wie denn in der That $3=1+2$ und $=-1+2.2$ ist.

Man kann jedoch den obigen Sätzen noch einige nähere Bestimmungen hinzufügen, die zwar an sich willkürlich sind, die aber nicht blofs bewirken, dafs auch die beiden ersten Primzahlen der allgemeinen 
Regel unterworfen sind, sondern auch in die Bildungsart der folgenden Primzahlen aus den vorhergehenden eine gewisse Einförmigkeit hineinbringen. Es ist nämlich zuvörderst offenbar, dafs nicht alle Primzahlen aus allen vorhergehenden durch blofse Addition und Substraction auf eine einzige Weise zu bilden seyen. So ist z. B.

und auch

$$
13=1+2-3-5+7+11 \text {, }
$$

$$
13=-1+2+3+5-7+11 \text {, }
$$

und namentlich wird dies zuerst bei jeder geradstelligen Primzahl Statt finden, die sich von der nächstvorhergehenden um 2 unterscheidet, und bei deren Zusammensetzung aus den kleineren Primzahlen 2 positiv genommen werden mufste. Denn da, wie sogleich genauer gezeigt werden wird, bei allen geradstelligen Primzahlen die nächstvorhergehende positiv genommen werden kann, so ist, wenn $M$ eine solche geradstellige, $L$ die nächstvorhergehende ungeradstellige Primzahl, und $M-L=2$ ist, $M$ von der Form

Setzt man nun

$$
M= \pm 1+2 \pm 3 \pm 5 \pm \ldots \pm K+L \text {. }
$$

so ist

$$
x=\mp 1+2 \mp 3 \mp 5 \mp \ldots . . . K+L \text {, }
$$

und folglich

$$
M+x=4+2 L
$$

$$
\boldsymbol{x}=M \text {, }
$$

so dafs also eine solche Primzahl aus allen vorhergehenden durch blofse Addition und Subtraction mindestens auf zwei verschiedene Weisen zusammengesetzt werden kann. Sodann aber sieht man auch, dafs bei jeder Primzahl, in deren Bildung die Form $1+2-3$ oder $2+P-Q$ vorkömmt, wo $P, Q$ zwei beliebige auf einander folgende Primzahlen vorstellen, deren Unterschied $=2$ ist, für diese Formen die ihnen resp. gleichgeltenden $-1-2+3$ oder $-2-P+Q$ gesetzt werden können. So ist z. B.

$$
\begin{aligned}
17 & =1+2-3-5+7-11+2.13, \\
& =-1-2+3-5+7-11+2.13, \\
& =-1+2+3+5-7-11+2.13,
\end{aligned}
$$

und auf dieselbe Art werden eine Menge, wo nicht die meisten Primzahlen auf mehrere verschiedene Weisen aus allen kleineren Primzahlen zusammenzusetzen sein. Um nun die A Anzahl dieser verschiedenen Bildungsarten zu beschränken, scheint es zweckmälsig, die Vorzeichen noch an 
ein besonderes Gesetz zu binden, welches sie erfüllen sollen. Wäre es eine Möglichkeit, ein solches zu finden, welches sich auf die A ufein a nderfolge der Vorzeichen bezöge, so wäre dies offenbar von der höchsten Wichtigkeit, da man hierdurch ein directes Mittel hätte, jede Primzahl aus den vorhergehenden zusammenzusetzen. Da sich mir jedoch kein solches darbot, so richtete ich mein Augenmerk zuerst nur auf die Anzahl der positiven und der negativen Glieder, und hier hat sich denn durch Beobachtung desjenigen Gesetzes, welches bei den Primzahlen Statt findet, die nur auf eine einzige Weise aus den vorhergehenden gebildet werden können, gleichfalls durch Induction gefunden, dafs es jedesmal möglich ist, bei der Bildung der geradstelligen Primzahlen die beiden nächtvorhergehenden positiv, von den andern aber gleich viele positiv und negativ, bei der Bildung der ungeradstelligen Primzahlen die nächstvorhergehende positiv, und, wie schon erwähnt, doppelt, von den anderen aber gleichfalls gleich viele positiv und negativ $\mathrm{zu}$ nehmen.

Aber auch jetzt ist die Bildungsart der Primzahlen noch nicht auf eine einzige eingeschränkt. Denn man sieht zum z. B., dals wenn $Q-P$ $=a_{1}, Q^{\prime}-P^{\prime}=a$ ist, für die Form $P-Q-P^{\prime}+Q^{\prime}$ die ihr gleichgeltende $-P+Q+P^{\prime}-Q^{\prime}$ gesetzt werden kann, ohne dafs die Anzahl der positiven und der negativen Glieder eine Veränderung erleidet. So ist z. B.

und auch

$$
23=1-2+3-5+\%+11-13-17+2.19
$$

$$
23=1-2+3+5-7-11+13-17+2.19 .
$$

Um also endlich zu einer einzigen Bildungsart zu gelangen, verfahre man auf folgende Weise. Soll eine geradstellige Primzahl aus allen vorhergehenden und der Zahl 1 durch blofse Addition und Subtraction zusammengesetzt werden, so nehme man zuerst 1 und alle kleineren geradstelligen Primzahlen positiv, alle kleineren ungeradstelligen aber negativ, mit Ausnahme der letzten ungeradstelligen Primzahl, welche positiv genommen werden muls; zieht man dann die zweite Summe von der ersten ab, so ist das Resultat jedesmal eine Zahl, welche grö [ser ist, als die zu bildende Primzahl (nur bei 3 und 7 ist es der zu bildenden Zahl gleich). Um nun den Unterschied des Resultats und der zu bildenden Primzahl, welcher nothwendig eine gerade $Z$ ahl ist, wegzuschaffen, versetze man so oft als nöthig eine positive Primzahl und eine kleinere negative mit 
einander, und man wird im Allg emeinen am schnellsten zum gewünschten Ziele gelangen, wenn man, mit Ausnahme der beiden grölsten positiv zu nehmenden Primzahlen, stets die möglichst gröfste positive mit einer möglichst kleinen negativen Zahl versetzt, immer jedoch das Princip festhält, durch keine Versetzung ein Resultat hervorzubringen, welches kleiner ist, als die verlangte Primzahl. Ganz auf dieselbe Weise wird jede ungeradstellige Primzahl gebildet, nur mit dem Unterschiede, dafs man gleich Anfangs blofs die einzige nächstvorhergehende Primzahl positiv, aber diese dann doppelt zu nehmen hat.

Beisp. 1. Es soll die 10te Primzahl 29 aus den vorherigen gebildet werden. Es ist

$$
\begin{aligned}
& 1-2 \\
& +3-5 \\
& +7-11 \\
& +13-17 \\
& +19 \\
& +23 \\
& \hline=66-35=31 .
\end{aligned}
$$

Demnach der Unterschied $31-29$ durch Versetzung wegzuschaffen, welches geschieht, wenn $+2-3$ statt $-2+3$ gesetzt wird.

2. Es soll die 17te Primzahl 59 aus den vorhergehenden gebildet werden. Es ist

$$
\begin{gathered}
\\
+1-2 \\
+2-5 \\
+13-11 \\
+19-23 \\
+29-31 \\
+37-41 \\
+43-47 \\
+2.53=+106 \\
\hline 258-177=81
\end{gathered}
$$

Folglich ist $81-59=22$ durch Versetzung wegzuschaffen. Da nun $43-11$ $=32$, durch Versetzung von $43 \mathrm{mit} 31$ also das Resultat kleiner als 59 würde, so kann man 43 nur mit 41 versetzen; eben so ist $37-9=26$, 
also mufs 37 mit 31 versetzt werden; $29-3=26$, also kann 29 nicht versetzt werden; $19-3=16$, folglich müssen 19 und 17 ihre Stellen vertauschen, und die noch übrigbleibende 1 wird durch Versetzung von 3 und 2 fortgeschafft, so dafs

$59=1+2-3-5+7-11+13+17-19-23+29+31-37+41-43-47+2.53$.

Nach diesen Regeln ist die diesem Hefte angefügte, mit II. bezeichnete Tafel berechnet, und bis zur Primzahl 499 ausgedehnt worden.

Diese Tafel enthält die Coefficienten, mit welchen die über denselben stehenden Primzahlen multiplicirt werden müssen, um die zu Anfang jeder Horizontalreihe als Argument stehende Primzahl zum Resultat zu geben, so dafs z. B. $11=+1-2+3-5+2.7$ u. s. w. Aufser den angegebenen habe ich noch die Primzahl 5003 nach denselben Gesetzen berechnet.

Ein Gesetz der Vorzeichen ist in dieser Tafel auf keine Weise zu erkennen; ein solches liefs sich aber auch bei der angegebenen durchaus willkürlichen Bildungsart gar nicht erwarten. Nur dies kann man bemerken: je weiter die zu bildende Primzahl hinaus liegt, eine desto geringere Anzahl von Primzahten hat man aus der ursprünglichen Anordnung zu versetzen, so dafs mit Ausnahme der Zahlen 2 und 3 , welche häufig ihr Vorzeichen vertauschen, die Vorzeichen immer mehr abwechselnd plus und minus sind. So ist z. B. für 5003 die Summe der ursprüuglichen positiven Glieder $=781969$, der negativen $=774388$, also ihr Unterschied 7581, demnach noch wegzuschaffen $7581-5003=2578$, welches durch die Versetzung von, 4973 mit 3691 , von 4937 mit 4933, von 4789 mit 4787 und von 3 mit 2 bewirkt wird, so dafs, mit Ausnahme vor 2 und 3, die Vorzeichen bis zu $36 \% 7$ abwechselnd positiv und negativ sind.

Eine sehr einfache Folgerung aus den aufgestellten Sätzen ist, dafs die natürliche Reihe der Primzahlen, wenn diese von 1 bis zu einer beliebigen hin, abwechselnd positiv und negativ genommen werden, ein Resultat giebt, welches grö fser ist (nur bei den 5 ersten Primzahlen ist es nicht gröfser, sondern gleich), als die nächstfolgende Primzahl, wobei jedoch zu bemerken ist, dafs die letzte Primzahl der Reihe positiv genommen werden mufs, wenn die vorhergehende positiv, und doppelt, wenn die vorhergehende negativ ist. So z. B. ist 
$2.1=2$

$$
\begin{aligned}
& 1+2=3 \\
& 1-2+2.3=5 \\
& 1-2+3+5=7 \\
& 1-2+3-5+2.7=11 \\
& 1-2+3-5+7+11>13 \\
& 1-2+3-5+7-11+2.13>17 \\
& 1-2+3-5+7-11+13+17>19 \\
& \text { etc. }
\end{aligned}
$$

Eine Bemerkung des Herrn Verfassers über Primzahlen, aus einem Briefe an den Herausgeber.

Die Primzahlen von der Form $4 n+1$ unterscheiden sich von denen $4 n+3$ auf so vielfache und entschiedene Weise, dafs man, da über das Bildungsgesetz der Primzahlen, vorausgesetzt, dafs ein solches überhaupt entweder für alle, oder nur für gewisse Gattungen der Primzahlen existirt, gar nichts bekannt ist, von vorn herein die Frage, ob bis zu einer gegebenen Zahl hin, gleichviel e Primzahlen $4 n+1$ und $4 n+3$ sich finden, wie ich glaube, nicht leicht entschieden wird bejahen, aber eben so wenig verneinen wollen. Der einzige Grund für eine bejahende Antwort müchte der sein, dafs man nicht absehe, weswegen in der Reihe aller ungeraden Zahlen, von der Form $4 n+1$, sich mehr oder weniger Primzahlen finden sollten, als in der Reihe aller ungeraden Zahlen $4 n+3$, da doch beide Reihen gleichviel Zahlen enthalten. Aber selbst dieșen Schlufs, dessen Schwäche übrigens einlẹuchtet, zugegeben, so schien es mir doch sicherer, einmal die Frage auf dem Wege der reinen Erfahrung, also durch blofses Abzählen bis zu einer nicht unbeträchtlichen Gränze hin, wenn auch nicht zur völligen, so doch zu einer A rt von Entscheidung zu bringen. Auf diese Weise hat sich ergeben, dafs die Primzahlen sich auf folgende Weise eintheilen. Es finden sich: 
13. Scherk, Bemerkungen über Primzahlen.

\begin{tabular}{|c|c|c|c|c|c|c|c|c|}
\hline \multirow{3}{*}{$\begin{array}{l}\text { bis } \\
1000\end{array}$} & \multicolumn{2}{|c|}{ Primzahlen } & \multirow{3}{*}{$\begin{array}{c}\text { bis } \\
18000\end{array}$} & \multirow{2}{*}{\multicolumn{2}{|c|}{$\begin{array}{c}\text { Primzahlen } \\
4 n+1 \mid 4 n+3\end{array}$}} & \multirow{3}{*}{$\begin{array}{c}\text { bis } \\
35000\end{array}$} & \multicolumn{2}{|c|}{ Primzahlen } \\
\hline & $4 n+1$ & $n+3$ & & & & & $4 n+$ & $4 n+3$ \\
\hline & 81 & 87 & & 1023 & 1041 & & 1865 & 1867 \\
\hline 2000 & 148 & 155 & 19000 & $10 \% 4$ & 1084 & 36000 & 1908 & 1916 \\
\hline 3000 & 212 & 218 & 20000 & 1131 & 1131. & 37000 & 1958 & 1964 \\
\hline 4000 & 269 & 281 & 21000 & 1178 & 1182 & 38000 & 2007 & 2010 \\
\hline 5000 & 331 & 338 & 22000 & 1229 & 1235 & 39000 & 2054 & 2053 \\
\hline 6000 & 385 & 398 & 23000 & 1278 & 1286 & 40000 & 2096 & 2107 \\
\hline 7000 & 444 & 456 & 24000 & 1332 & 1336 & 41000 & 2138 & 2153 \\
\hline 8000 & 501 & 506 & 25000 & 1377 & 1385 & 42000 & 2190 & 2202 \\
\hline 9000 & 556 & 561 & 26000 & 1428 & 1432 & 43000 & 2244 & 2250 \\
\hline 10000 & 611 & 618 & 27000 & 1484 & 1477 & 44000 & 2288 & 2291 \\
\hline 11000 & 661 & 674 & 28000 & 1527 & 1528 & 45000 & 2335 & 2340 \\
\hline 12000 & 710 & 728 & 29000 & 1574 & $15 \% 9$ & 46000 & 2384 & 2377 \\
\hline 13000 & 769 & 778 & 30000 & 1618 & $162 \%$ & 47000 & 2326 & 2325 \\
\hline 14000 & 821 & 831 & 31000 & 1670 & 1670 & 48000 & 2476 & 2470 \\
\hline 15000 & 869 & 885 & 32000 & 1714 & 1718 & 49000 & 2520 & 2515 \\
\hline 16000 & 923 & 939 & 33000 & 1769 & 1769 & 50000 & 2566 & 2567 \\
\hline 17000 & 972 & 988 & 34000 & 1822 & 1816 & & & \\
\hline
\end{tabular}

Hieraus geht also, wie zu erwarten war, hervor, dals man mit groIser Wahrscheinlichkeit annehmen kann, die Anzahl der Primzahlen $4 n+1$ sei der Anzahl der Primzahlen $4 n+3$ nicht blols im Allgemeinen, sondern auch bis zu einer gegebenen Grenze hin sehr nahe gleich. 